\title{
Charge identification of nuclear fragments with the FOOT Time-Of-Flight system
}

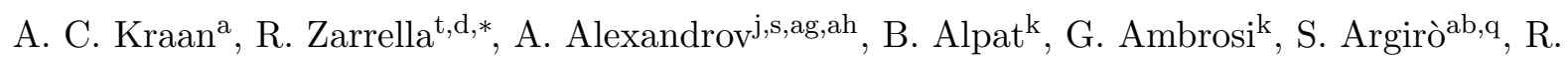
Arteche Diaz $^{\text {ad }}$, N. Bartosik ${ }^{\mathrm{q}}$, G. Battistoni ${ }^{\mathrm{h}}$, N. Belcari ${ }^{\mathrm{b}, \mathrm{a}}$, E. Bellinzona ${ }^{\mathrm{o}}, \mathrm{S}$. Biondi ${ }^{\mathrm{d}, \mathrm{t}}, \mathrm{G}$. Bruni $^{\mathrm{d}}$, P. Carra ${ }^{\mathrm{b}, \mathrm{a}}$, P. Cerello ${ }^{\mathrm{q}}$, E. Ciarrocchi ${ }^{\mathrm{b}, \mathrm{a}}$, A. Clozza ${ }^{\mathrm{g}}$, S. Colombi ${ }^{\mathrm{o}, \mathrm{p}}$, G. De Lellis ${ }^{\mathrm{s}, \mathrm{j}}$, A. Del Guerra ${ }^{\mathrm{b}, \mathrm{a}}$, M. De Simoni ${ }^{\mathrm{l}, \mathrm{z}}$, A. Di Crescenzo ${ }^{\mathrm{s}, \mathrm{j}}$, B. Di Ruzza ${ }^{\circ}$, M. Donetti $^{\mathrm{e}}$, Y. Dong ${ }^{\mathrm{h}, \mathrm{w}}, \mathrm{M}$. Durante $^{f, a f}$, R. Faccini ${ }^{z, l}$, V. Ferrero ${ }^{q}$, E. Fiandrini ${ }^{k, x}$, C. Finck ${ }^{n}$, E. Fiorina ${ }^{q}$, M. Fischetti ${ }^{1, v}$, M. Francesconi ${ }^{\mathrm{b}, \mathrm{a}}$, M. Franchini ${ }^{\mathrm{d}, \mathrm{t}}$, G. Franciosini ${ }^{\mathrm{l}, \mathrm{z}}$, G. Galati ${ }^{\mathrm{j}}$, L. Galli $^{\mathrm{a}}$, V. Gentile ${ }^{\mathrm{j}}, \mathrm{ag}$, G.

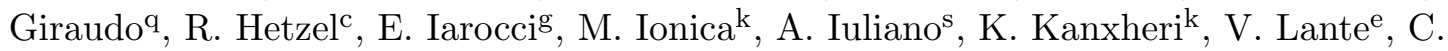

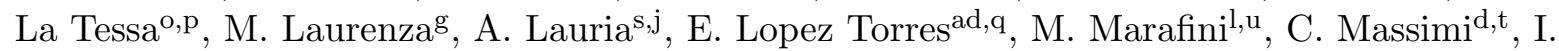

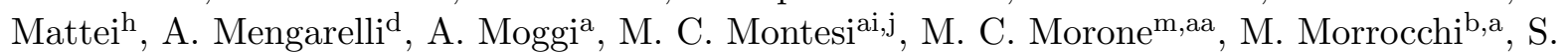

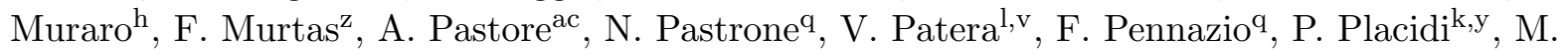
Pullia $^{\mathrm{e}}$, F. Raffaellii ${ }^{\mathrm{a}}$, L. Ramello ${ }^{\mathrm{r}, \mathrm{q}}$, R. Ridolfi, ${ }^{\mathrm{d}, \mathrm{t}}$, V. Rosso ${ }^{\mathrm{b}, \mathrm{a}}$, C. Sanelli ${ }^{\mathrm{g}}$, A. Sarti ${ }^{\mathrm{l}, \mathrm{v}}$, G.

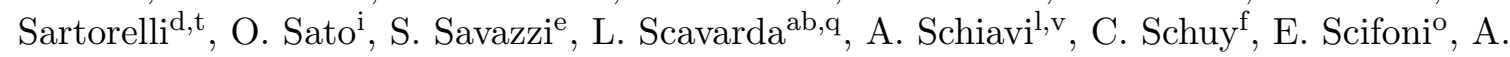

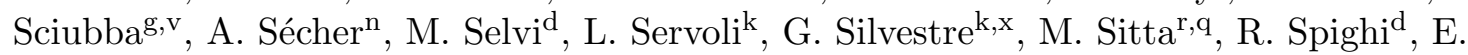

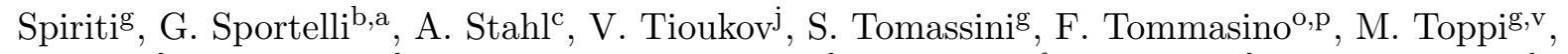
G. Traini ${ }^{\mathrm{l}, \mathrm{z}}$, S. M. Valle ${ }^{\mathrm{h}}$, M. Vanstalle ${ }^{\mathrm{n}}$, M. Villa ${ }^{\mathrm{d}, \mathrm{t}}$, U. Weber ${ }^{\mathrm{f}}$, A. Zoccoli ${ }^{\mathrm{d}, \mathrm{t}}$, M. G. Bisogni ${ }^{\mathrm{b}, \mathrm{a}}$

${ }^{a}$ Istituto Nazionale di Fisica Nucleare (INFN), Section of Pisa, Pisa, Italy

${ }^{b}$ University of Pisa, Department of Physics, Pisa, Italy

${ }^{c}$ RWTH Aachen University, Physics Institute III B, Aachen, Germany

${ }^{d}$ Istituto Nazionale di Fisica Nucleare (INFN), Section of Bologna, Bologna, Italy

${ }^{e}$ Centro Nazionale di Adroterapia Oncologica (CNAO), Pavia, Italy

${ }^{f}$ Biophysics Department, GSI Helmholtzzentrum für Schwerionenforschung, Darmstadt, Germany

${ }^{g}$ Istituto Nazionale di Fisica Nucleare (INFN), Laboratori Nazionali di Frascati, Frascati, Italy

${ }^{h}$ Istituto Nazionale di Fisica Nucleare (INFN), Section of Milano, Milano, Italy ${ }^{i}$ Nagoya University, Department of Physics, Nagoya, Japan

${ }^{j}$ Istituto Nazionale di Fisica Nucleare (INFN), Section of Napoli, Napoli, Italy

${ }^{k}$ Istituto Nazionale di Fisica Nucleare (INFN), Section of Perugia, Perugia, Italy

${ }^{l}$ Istituto Nazionale di Fisica Nucleare (INFN), Section of Roma 1, Rome, Italy

${ }^{m}$ University of Rome Tor Vergata, Department of Physics, Rome, Italy

${ }^{n}$ Université de Strasbourg, CNRS, IPHC UMR 7871, F-67000 Strasbourg, France

${ }^{\circ}$ Trento Institute for Fundamental Physics and Applications, Istituto Nazionale di Fisica Nucleare (TIFPA-INFN), Trento, Italy

${ }^{p}$ University of Trento, Department of Physics, Trento, Italy

${ }^{q}$ Istituto Nazionale di Fisica Nucleare (INFN), Section of Torino, Torino, Italy

${ }^{r}$ University of Piemonte Orientale, Department of Science and Technological Innovation, Alessandria, Italy

${ }^{s}$ University of Napoli, Department of Physics "E. Pancini", Napoli, Italy

${ }^{t}$ University of Bologna, Department of Physics and Astronomy, Bologna, Italy

${ }^{u}$ Museo Storico della Fisica e Centro Studi e Ricerche Enrico Fermi, Rome, Italy

${ }^{v}$ University of Rome La Sapienza, Department of Scienze di Base e Applicate per l'Ingegneria (SBAI), Rome, Italy

${ }^{w}$ University of Milano, Department of Physics, Milano, Italy

${ }^{x}$ University of Perugia, Department of Physics and Geology, Perugia, Italy

${ }^{y}$ University of Perugia, Department of Engineering, Perugia, Italy

${ }^{z}$ University of Rome La Sapienza, Department of Physics, Rome, Italy

${ }^{a}$ Istituto Nazionale di Fisica Nucleare (INFN), Section of Roma Tor Vergata, Rome, Italy

${ }^{a b}$ University of Torino, Department of Physics, Torino, Italy

${ }^{a c}$ Istituto Nazionale di Fisica Nucleare (INFN), Section of Bari, Bari, Italy

${ }^{a d}$ CEADEN, Centro de Aplicaciones Tecnologicas y Desarrollo Nuclear, Havana, Cuba

${ }^{a e}$ Gran Sasso Science Institute, L'Aquila, Italy

${ }^{a f}$ Technische Universität Darmstadt Institut für Festkörperphysik, Darmstadt, Germany

${ }^{a g}$ National University of Science and Technology, MISIS, RUS-119049 Moscow, Russia

\footnotetext{
${ }^{*}$ Corresponding author. Formerly at University of Pisa and INFN, Section of Pisa
}

Email address: roberto.zarrella2@unibo.it (R. Zarrella) 
${ }^{a h}$ Lebedev Physical Institute of the Russian Academy of Sciences, RUS-119991 Moscow, Russia

${ }^{a i}$ University of Napoli, Department of Chemistry, Napoli, Italy

\begin{abstract}
FOOT (FragmentatiOn Of Target) is an applied nuclear physics experiment designed for measuring with high precision the production cross sections of nuclear fragments for energies, beams and targets relevant in particle therapy and radioprotection in space. These measurements are important to estimate the physical and biological effects of nuclear fragments, which are produced when energetic particle beams penetrate human tissue.

A component of the FOOT experiment is the $\triangle E$-TOF system, which is designed to measure energy loss and time-of-flight of nuclear fragments produced in particle collisions in thin targets in order to extract their charge and velocity. The $\Delta E$-TOF system is composed of a start counter, providing the start time for the time-of-flight, and a $40 \times 40 \mathrm{~cm}^{2}$ wall of thin plastic scintillator bars, providing the stop time and energy loss of the fragments passing through the detector. Particle charge discrimination can be achieved by correlating the energy loss in the scintillator bars with the measured time-of-flight.

Recently, we have built a full-scale $\Delta E$-TOF detector prototype. In this work, we describe the energy and time-of-flight calibration procedure and assess the performance of the $\Delta E$-TOF prototype. We use data acquired during beam tests at $\mathrm{CNAO}$ with proton and ${ }^{12} \mathrm{C}$ beams and at GSI with ${ }^{16} \mathrm{O}$ beams in the energy range relevant for particle therapy, from 60 to 400 $\mathrm{MeV} / \mathrm{u}$. For heavy fragments $(\mathrm{C}$ and $\mathrm{O}$ ), we obtain energy and time resolutions ranging from 4.0 to $5.2 \%$ and from 54 to $84 \mathrm{ps}$, respectively, The procedure is also applied to a fragmentation measurement of a $400 \mathrm{MeV} / \mathrm{u}^{16} \mathrm{O}$ beam on a $5 \mathrm{~mm}$ carbon target, showing that the system is capable of discriminating the charges of impinging fragments.
\end{abstract}

Keywords: FOOT, charge identification, nuclear fragmentation, particle therapy, time-of-flight

\title{
1. Introduction
}

Particle therapy is an external beam radiotherapy technique that uses charged particles (mostly protons or ${ }^{12} \mathrm{C}$ ions) for tumor treatment. Thanks to their typical depth-dose profile (Bragg peak), more conformal dose distributions can be realized with charged particle therapy than with conventional radiotherapy [1]. Although particle therapy is becoming a more widespread treatment modality, much research is still needed to further improve its accuracy.

An important research topic is to reduce the uncertainty of the radiobiological effectiveness (RBE) of particle beams. This quantity, needed to calculate biological dose and to predict treatment outcomes, demonstrates a complex dependency on several physical and biological parameters, among which the spectrum of nuclear fragments produced in interactions of the particle beam with the patient tissue [2-4]. To simulate the biological effect of these fragments and take it into account in particle therapy treatment planning, it is important to accurately model their production $[5,6]$. Differential cross section measurements for nuclear fragment production in thin targets are the most valuable for this purpose. However, such data are scarce and limited to few projectiles and energies [7-10]. Apart from particle therapy, these measurements are also of great importance for radioprotection in space [11]. 
The goal of the FOOT (FragmentatiOn Of Target) experiment [12] is to provide double differential cross section measurements with respect to kinetic energy and direction for beams and targets of interest for particle therapy. Using a fixed target setup, all relevant characteristics of fragments produced in thin tissue-like targets will be measured for a variety of particle beams. FOOT aims at achieving a resolution of approximately $2-3 \%$ and $5 \%$ for charge and mass measurements of nuclear fragments, respectively, and a $5 \%$ accuracy on cross section measurements [13]. To reach this goal, FOOT will acquire data with two different setups: an emulsion spectrometer, dedicated to the detection of light charged fragments $(Z \leq 3)$, and an electronic setup, focused on the study of nuclei with $3 \leq Z \leq 8$, defined here as 'heavy'. A full description of both setups is reported in $[13,14]$.

A key component in the electronic setup is the $\Delta E$-TOF system, which will derive the charge and velocity of the fragments through energy loss and time-of-flight (TOF) measurements. A new full-scale prototype was recently constructed, that includes a start counter and a $40 \times 40$ $\mathrm{cm}^{2}$ wall of thin plastic scintillator bars.

The goal of the present work is to assess the performance of the full scale $\Delta E$-TOF prototype in terms of TOF and $\Delta E$ accuracy, and to verify whether the FOOT requirements are met, using new beam test measurements at two different particle therapy treatment centers. In particular, we describe an energy and time calibration procedure to discriminate fragment charges, we validate it, and apply it to detect the fragments produced in nuclear interactions of a 400 $\mathrm{MeV} / \mathrm{u}^{16} \mathrm{O}$ beam impinging on a $5 \mathrm{~mm}$ graphite target. The measurements performed are the first tests of this full prototype.

\section{Materials and methods}

\subsection{Detectors and DAQ system}

The aim of the $\Delta E$-TOF system is to identify the charge of impinging particles with a resolution of the order of 2-3\% for heavy nuclei, such as C, N, and O. To reach this goal the system should achieve for such nuclei an accuracy in energy loss and TOF measurements of at most $5 \%$ and 100 ps respectively $[13,14]$.

The $\Delta E$-TOF prototype consists of two scintillation detectors: the Start Counter (SC) and the TOF-Wall (TW). The SC, the first detector encountered by the beam, is a thin $(250 \mu \mathrm{m})$ foil of plastic scintillator (EJ-228, Eljen Technology). The detector has an active area of $5 \times 5$ $\mathrm{cm}^{2}$ and its main purpose is to provide the rate of primaries and the start time of each event. The light produced in the SC is collected at each side of the foil using a total of $483 \times 3 \mathrm{~mm}^{2}$ ASD-NUV3S SiPMs with $25 \mu \mathrm{m}$ microcell pitch [15].

The TW is made of 40 bars of plastic scintillator (EJ-200, Eljen Technology) arranged in two orthogonal layers of 20 each. Each bar has an active area of $44 \times 2 \mathrm{~cm}^{2}$ and is $3 \mathrm{~mm}$ thick. The light produced in the bars is collected at each end by four $3 \times 3 \mathrm{~mm}^{2}$ MPPC SiPMs with 25 $\mu \mathrm{m}$ microcell pitch. The purpose of the TW is to measure the energy loss $\Delta E$ of the particles and to provide the stop time for TOF measurements. The characterisation and performance of the bars was described previously [16-19]. Figure 1 shows a picture of the two components of the $\Delta E$-TOF system.

The signals collected by the SiPMs of both detectors are sampled through WaveDREAM digitizer boards (WDB), integrated in the WaveDAQ system [20-22]. The WDBs can sample up to 16 channels and also provide the supply voltage to the SiPMs of the detectors. The readout of the $\Delta E$-TOF employs 8 independent analog channels (connected to $1 \mathrm{WDB}$ ) for the SC and 


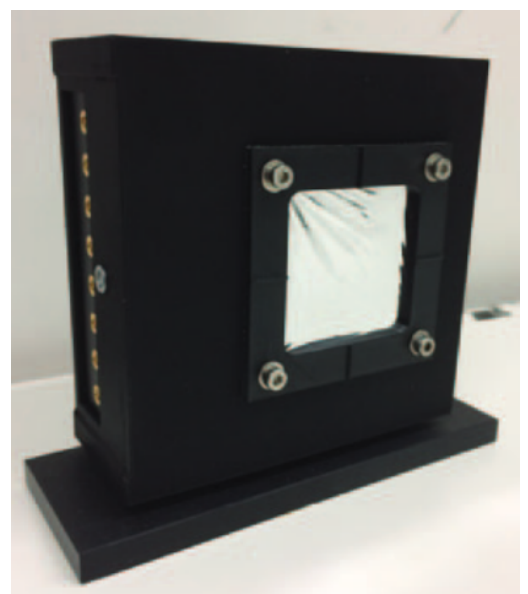

(a)

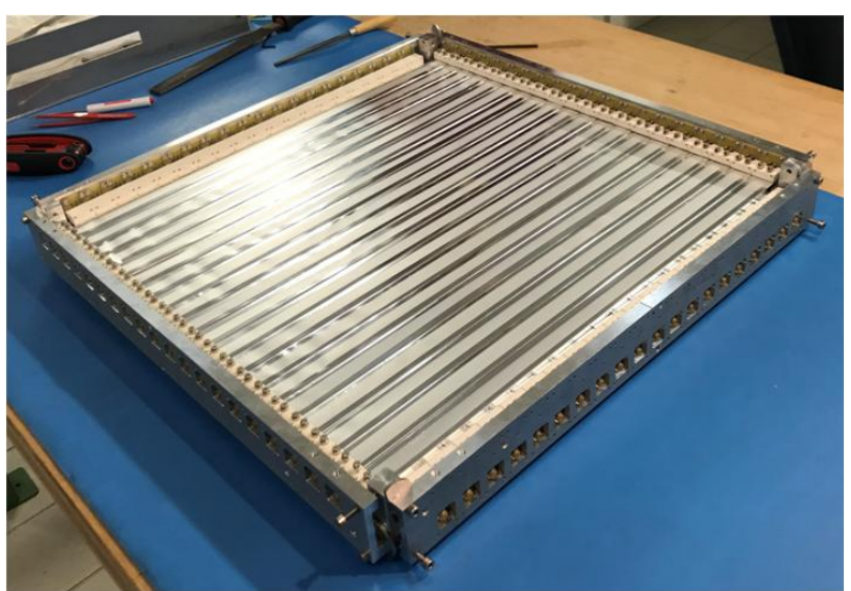

(b)

Figure 1: Components of the $\Delta E$-TOF system: (a) Start Counter and (b) TOF-Wall.

Table 1: Summary of the data samples analyzed in this work: treatment center, particle beam, energy, purpose of the acquisition, and target (if present).

\begin{tabular}{|c|c|c|c|c|}
\hline Setup & Particle & Energy $[\mathrm{MeV} / \mathrm{u}]$ & Purpose & Target \\
\hline CNAO & $\mathrm{p}$ & 60 & Calibration & - \\
CNAO & ${ }^{12} \mathrm{C}$ & 115 & Calibration & - \\
CNAO & ${ }^{12} \mathrm{C}$ & 260 & Calibration & - \\
CNAO & ${ }^{12} \mathrm{C}$ & 400 & Calibration & - \\
\hline GSI & ${ }^{16} \mathrm{O}$ & 400 & Calibration & - \\
GSI & ${ }^{16} \mathrm{O}$ & 400 & Fragmentation & 5 mm graphite \\
\hline
\end{tabular}

2 channels for each bar of the TW ( 80 channels and 7 WDBs in total). The data acquisitions considered in this work were performed with a sampling frequency of 4 GSamples/s.

\subsection{Data samples}

Data were acquired during two beam tests performed recently. A summary of the irradiations is given in Table 1. The first beam test was carried out at the Centro Nazionale di Adroterapia Oncologica (CNAO) in Pavia (Italy). The aim was to perform a set of detector calibration runs. The distance between $\mathrm{SC}$ and TW was $42.5 \mathrm{~cm}$. The beams employed were protons at $60 \mathrm{MeV}$ and ${ }^{12} \mathrm{C}$ ions at 115,260 and $400 \mathrm{MeV} / \mathrm{u}$. Due to time constraints, the TW was only partly irradiated. We ensured that all bars were irradiated at least at the center. The irradiation of the TW in different positions was carried out by moving the detector while keeping the beam line fixed. In this way, the distance between the SC and TW along the beam line was constant during the acquisitions. Events were recorded when at least one bar in each layer was triggered. A total of 5000 events was acquired in each irradiated TW hit position, defined as the region where two bars (one per layer) overlap (Fig. 2). Hereafter, hit positions will be denoted by the index $i$.

The second beam test was carried out at the GSI Heavy Ion Research Center in Darmstadt (Germany), two weeks after the CNAO data acquisitions. It should be noted that, between the two data takings, the $\Delta E$-TOF system was disassembled for transport and subsequently rebuilt, partly with different cables. In this case, the SC and TW were placed at a distance of $2.23 \mathrm{~m}$. The FOOT beam monitoring system, the vertex detector and a calorimeter module were also 


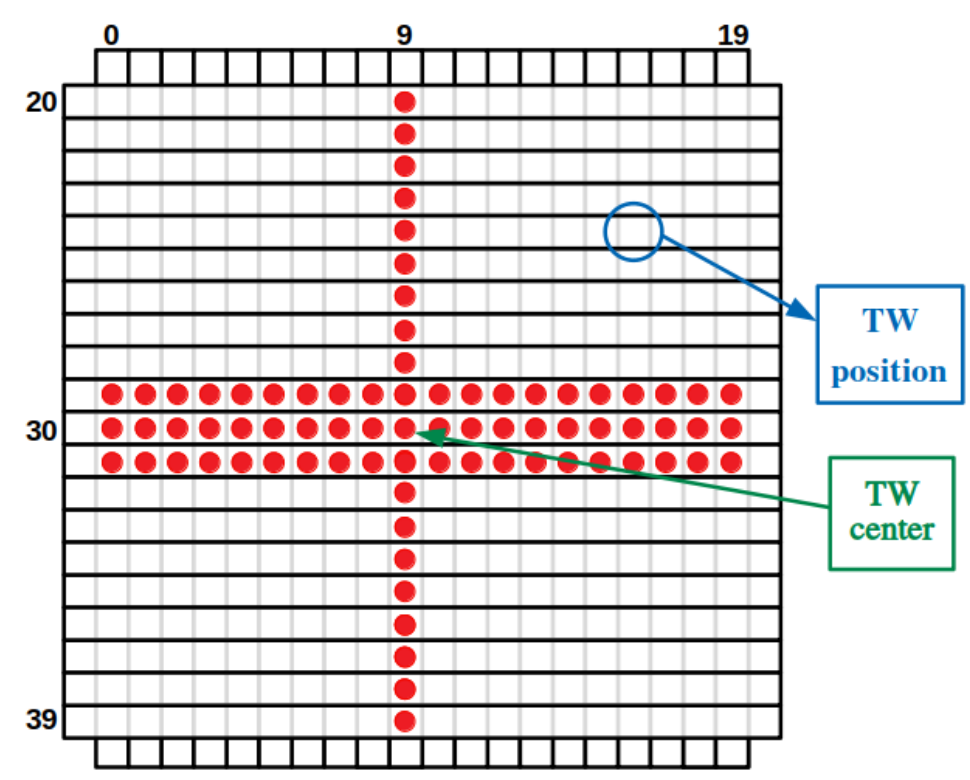

Figure 2: Schematic view of the TW showing the bar labels and the irradiated positions (red dots) during the calibration runs at CNAO.

present, but not included in this analysis. At GSI, we acquired two data samples. First, a calibration run was carried out with a ${ }^{16} \mathrm{O}$ beam at $400 \mathrm{MeV} / \mathrm{u}$ in the same way as described above. During this run, we acquired a total of $\sim 6.8 \cdot 10^{4}$ events in different TW positions. Due to time constraints, again only a limited number of bars was irradiated, and mostly in central positions. Second, we inserted a $5 \mathrm{~mm}$ graphite target to study the fragmentation of a 400 $\mathrm{MeV} / \mathrm{u}^{16} \mathrm{O}$ beam. Here the beam line was directed at the center of the TW and we acquired a total of $\sim 4.5 \cdot 10^{4}$ events. The setup of the data taking is shown in Fig. 3.

\section{3. $M C$ simulations}

We performed MC simulations in order to provide a set of reference values for the $\Delta E$ and TOF calibration procedures. The data samples of Table 1 were all simulated with the FLUKA code $[23,24]$ by accurately reproducing both setups. The simulations did not take into consideration light generation and optical photon transport inside the bars. For each data sample, $10^{6}$ events were simulated. For each event, we registered the interaction times of the particles with both detectors and the energy loss in the TW bars. We extracted for each event:

- The hit position $i$ of each particle passing through the bar;

- The time of flight $\mathrm{TOF}_{\mathrm{MC}, i, l}$ between SC and TW layer $l$, where $l=F$ for the front and $l=R$ for the rear layer, respectively;

- The energy loss $\Delta E_{\mathrm{MC}, i, l}$ in TW layer $l$;

For the fragmentation sample, we also registered the time it takes the primary ${ }^{16} \mathrm{O}$ beam to travel from the SC to the target, $\mathrm{TOF}_{\mathrm{MC}}^{\prime}$.

Two sets of MC simulations were produced. An initial set was produced, where only the intrinsic statistical fluctuations were included, and used to extract the reference values for the calibration (see Sec. 2.4 and 2.6). Furthermore a second set was produced after the calibration was applied, that included the observed average energy and time resolutions by means of 


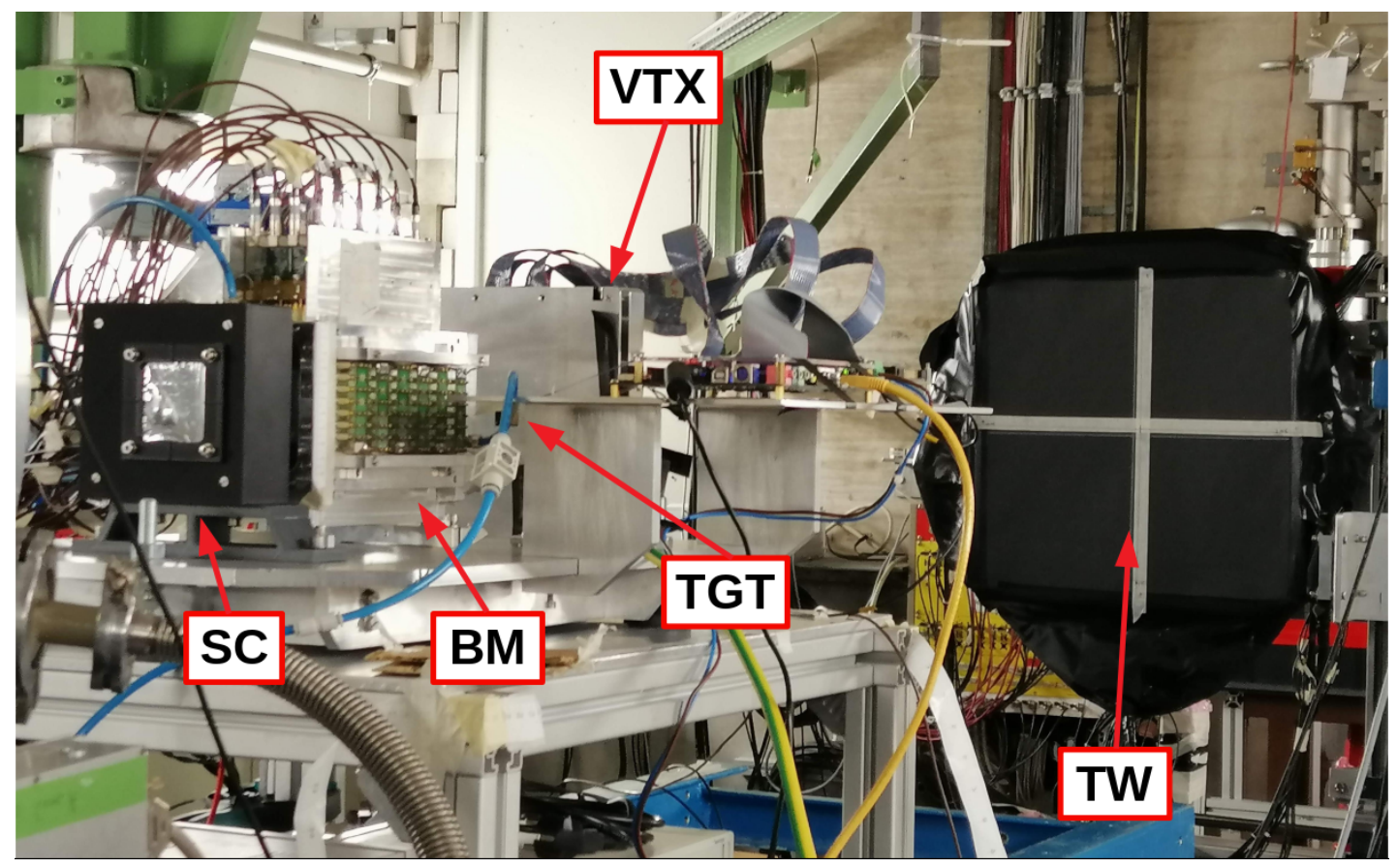

(a)

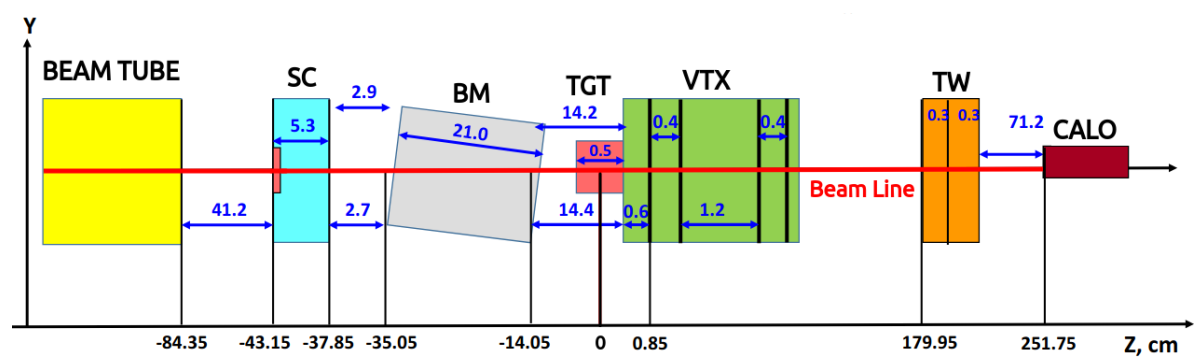

(b)

Figure 3: (a) Schematic view and (b) picture of the GSI setup. The pictures also show the target (TGT), the tracking detectors included in the setup, i.e. the Beam Monitor (BM) and the Vertex (VTX), and a calorimeter module (CALO). For a complete description of these components of FOOT, see [12, 13].

Gaussian smearing. These two sets are referred to as 'untuned' and 'tuned' MC simulations, respectively.

\section{4. $\Delta E$ calibration}

A signal processing routine was developed to handle the waveforms obtained from the SC and TW and all the associated WaveDAQ clocks (CLKs), so the raw energy loss and time-offlight could be extracted. We use the term 'raw' to indicate that both quantities still need to be calibrated to match $\mathrm{MC}$ values.

Regarding the energy loss, the raw values were extracted directly from the TW signals. First, the two channels of each bar involved in the event (hereafter indicated with the subscripts $A / B$ ) were processed separately and the charge collected in each of them, $Q_{A / B}$, was evaluated as the area of the signals. Supposing a simple exponential attenuation along the bar, the total charge collected was defined as [16]

$$
Q=\sqrt{Q_{A} \cdot Q_{B}}
$$


$Q$ represents the raw energy loss of particles traveling through the bar. Ideally, it should be independent of the hit position along the bar. However, we noticed that the mean value of $Q$, obtained by fitting with a Gaussian, varies along the bars, as can be seen in Fig. 4 for ${ }^{12} \mathrm{C}$ ions of $260 \mathrm{MeV} / \mathrm{u}$. Differences up to $15 \%$ were seen in this case. Moreover, the mean value of the detected charge was asymmetric for the two sides of the bar. We verified that other bars and data samples showed a similar trend. This may be due to differences in total internal reflection efficiency, light absorption along the bar, optical coupling and SiPM gain between the two sides. We come back to these issues in Sec. 4. A practical and accurate approach to take into account

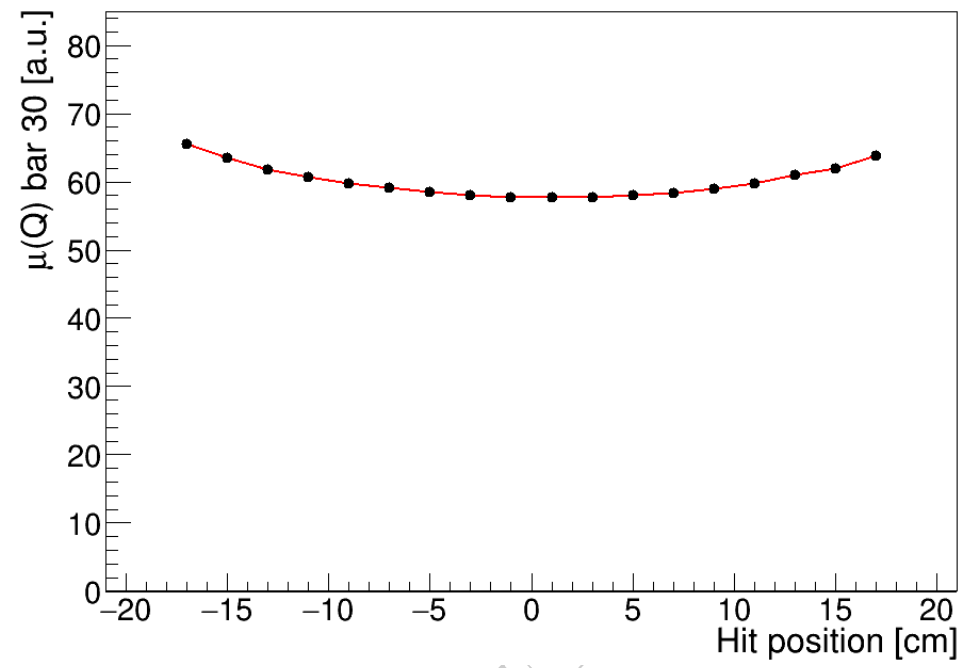

Figure 4: Mean value of the total charge collected $Q$ in the central bar of the front layer of the TW as a function of the hit position during the calibration run with $260 \mathrm{MeV} / \mathrm{u}{ }^{12} \mathrm{C}$ ions. Error bars are displayed but small, since they represent the standard error on the mean obtained from the Gaussian fit. The origin of the $\mathrm{x}$ axis indicates the center of the bar.

the observed position-dependence was to calibrate each TW position separately for the two TW layers. For each layer, we have $20 \times 20=400$ hit positions (see Fig. 2) to calibrate.

The method used to calibrate the charge response was based on finding a relationship between the detected charge and a reference energy loss value, which was obtained from the MC simulations. For this purpose the mean value $\mu\left(Q_{i, l}\right)$ of the collected charge $Q$ over all events in position $i$ in layer $l$ was plotted as a function of the mean energy loss expected from MC, $\mu\left(\Delta E_{\mathrm{MC}, i, l}\right)$, and fitted with Birks' model [25]:

$$
\mu\left(Q_{i, l}\right)=\frac{p_{0, i, l} \mu\left(\Delta E_{\mathrm{MC}, i, l}\right)}{1+p_{1, i, l} \mu\left(\Delta E_{\mathrm{MC}, i, l}\right)}
$$

where $p_{0, i, l}$ and $p_{1, i, l}$ represent respectively the charge conversion factor and the saturation parameter of the model. Although we applied this formula position-per-position, we do not believe that the true Birks' parameters $p_{0}$ and $p_{1}$ change along the bar. However, this approach helps to parameterise experimentally non trivial dependencies that cannot be described via the current MC simulations. In other words, we use Birks' model merely as a convenient parameterization.

Figure 5 displays an example of the energy calibration curve obtained for a position of the front layer of the TW. What can be seen is that the light output of the bar is reproduced by the simple model from Eq. 2. To assure a reliable fit, for each layer we applied Eq. 2 to all positions that registered more than 40 events in, at least, 4 of the 5 calibration samples. If this condition was satisfied in both layers of the TW, the position was defined as 'well-calibrated'. 


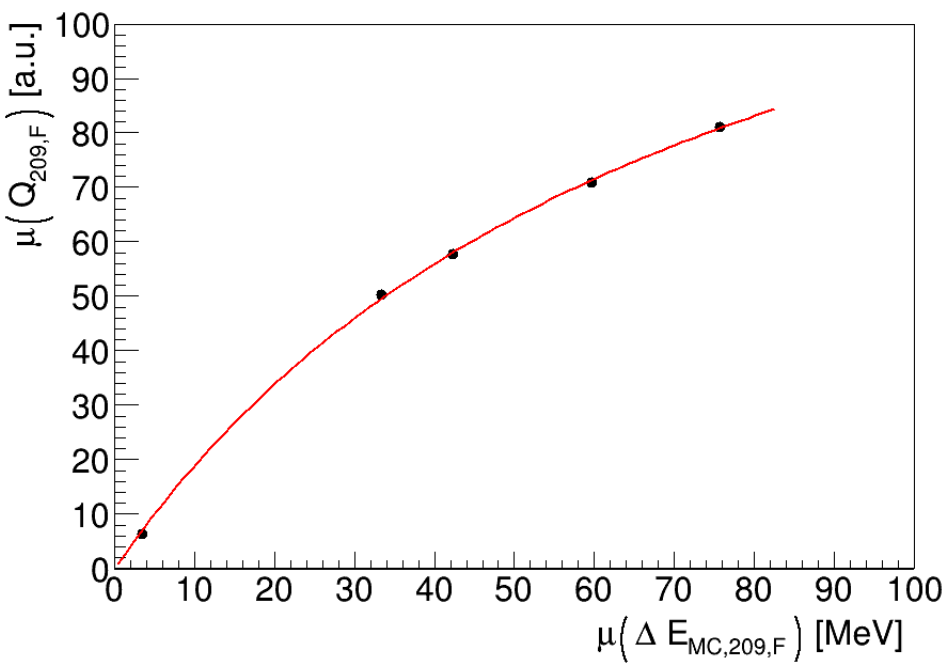

Figure 5: Energy calibration curve obtained with the Birks-like model for the central position $(i=209)$ of the front layer $(l=F)$ of the TW. Error bars are displayed but small, since they represent the standard error on the mean obtained from the Gaussian fit.

Using the parameters extracted through Eq. 2 for these positions, it was then possible to retrieve the energy loss of each event in a given position and layer through the inverse formula

$$
\Delta E_{i, l}=\frac{Q_{i, l}}{p_{0, i, l}-p_{1, i, l} Q_{i, l}}
$$

The calibrated energy loss of a particle in a given position $i, \Delta E_{i}$, was then defined as the sum of the energy loss in the two layers of the TW:

$$
\Delta E_{i}=\Delta E_{i, F}+\Delta E_{i, R}
$$

\subsection{Energy resolution}

The overall energy resolution of the system was then extracted from the calibrated energy loss values as follows. The $\Delta E_{i}$ (Eq. 4) values obtained for each event in all well-calibrated positions of a calibration sample were merged into one 'global' distribution to obtain the energy spectrum of the acquisition. Then, we performed a Gaussian fit on the resulting distribution to extract the mean value $\mu(\Delta E)$ and standard deviation $\sigma(\Delta E)$ of each sample. The energy resolution of the system was defined as the $\sigma(\Delta E)$ value obtained.

In order to perform a more realistic MC-data comparison, we needed to parameterize the contribution to the energy resolution of all the effects not described in detail in the MC, such as photon production, optical transport, electronics, etc. This contribution, indicated as $\sigma_{p a r}(\Delta E)$, could be disentangled by quadratically subtracting the intrinsic term, evaluated as the spread in untuned MC simulations, from the overall measured energy resolution $\sigma(\Delta E)$ in each calibration sample. We then parameterized the quantity $\sigma_{p a r}(\Delta E) / \mu(\Delta E)$ as a function of $\mu(\Delta E)$ with a constant and used this parameterization to produce the tuned MC simulations (see Sec. 2.3). Since data were acquired with two experimental setups under different conditions, the measured resolution may vary between the CNAO and GSI samples. Therefore, we chose to parameterize the response of the $\Delta E$-TOF only with CNAO data. The obtained results are discussed in Sec. 3.1. 
The start time $T_{S C}$ of each event was extracted from the SC waveforms. First, the 8 signals of the SC were summed into a single one. Then the value of $T_{S C}$ was obtained by applying a Constant Fraction Discriminator (CFD) algorithm to the summed waveform induced by the particle that passes through the detector. The fraction of the CFD was set to 0.3 on the basis of former studies [16].

The stop time of each event was extracted from the TW signals. For this purpose, the two channels of each TW bar involved in the event were processed separately and the raw TOF was extracted as follows. First, the single channel stop times $\left(T_{A / B}\right)$ were obtained through the CFD method, again with fraction 0.3. Second, the CLK signals between TW and SC had to be synchronized. This was achieved by calculating the phase of each CLK involved in the events and extracting the phase difference $\Delta_{C L K, A / B}$ between the clocks of the TW and that of the $\mathrm{SC}[26]$. Then, the raw TOF value was calculated for each bar as:

$$
\mathrm{TOF}_{\text {raw }}=T_{b a r}-T_{S C}
$$

where $T_{b a r}$ is the stop time associated to a TW bar, synchronized with the SC:

$$
T_{b a r}=\frac{T_{A}-\Delta_{C L K, A}+T_{B}-\Delta_{C L K, B}}{2}
$$

An example of the mean value of $\mathrm{TOF}_{\text {raw }}$ (obtained by fitting with a Gaussian) as a function of the hit position along one of the front bars is given in Fig. 6. Variations up to about 100 ps were found, with lower $\mathrm{TOF}_{\text {raw }}$ values at the sides of the bar (see Fig. 6). We believe that this effect originates from the same factors causing the charge variation (see Sec. 2.4). Therefore we calibrated the TOF taking into account the position where the bar was hit, as already done for the energy calibration.

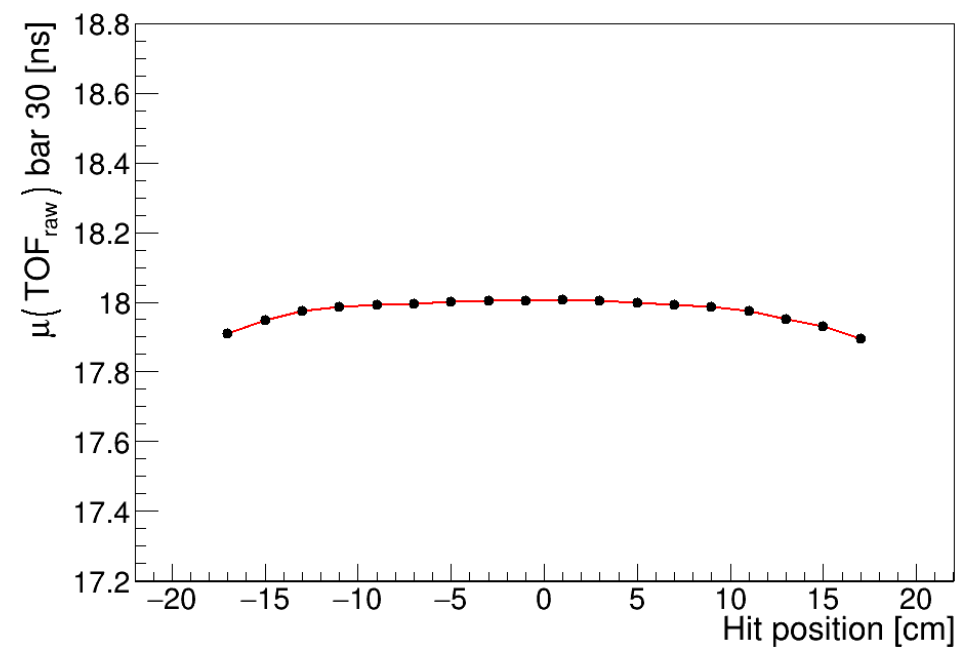

Figure 6: Mean value of the raw TOF measured along the central bar of the front TW layer for the ${ }^{12} \mathrm{C}$ beam at $260 \mathrm{MeV} / \mathrm{u}$. The origin of the $\mathrm{x}$ axis indicates the center of the bar, as in Fig. 4. Again, error bars are displayed but small, since they represent the standard error on the mean obtained from the Gaussian fit.

The TOF calibration was aimed at relating the raw TOF values from Eq. 5 for the calibration runs to a reference value, which we defined to be the expected TOF value between the SC to the TW, obtained from the MC simulations. For a given position $i$, we calculated for each layer $l$ the difference between the mean values of the data and $\mathrm{MC}$ distributions:

$$
\mu\left(\mathrm{TOF}_{\mathrm{raw}, i, l}\right)=\mu\left(\mathrm{TOF}_{\mathrm{MC}, i, l}\right)+a_{i, l}
$$


The variable $a_{i, l}$ accounts for all the possible time offsets, such as signal propagation through cables and global time shifts between the SC and TW.

With the extracted $a_{i, l}$ values, the calibrated TOF of each event in a given position and layer, $\mathrm{TOF}_{i, l}$, was retrieved by simply inverting Eq. 7:

$$
\mathrm{TOF}_{i, l}=\mathrm{TOF}_{\mathrm{raw}, i, l}-a_{i, l}
$$

For the fragmentation run, where fragments were produced in the target (which is located in between the SC and the TW, see Fig. 3a), the calibrated TOF values of the fragments should be calculated from target to TW rather than from SC to TW. In that case, we subtracted an additional offset $\mathrm{TOF}_{\mathrm{MC}}^{\prime}$ accounting for the time needed by the ${ }^{16} \mathrm{O}$ ions to travel from the SC to the target. In other words, for the fragmentation run we applied:

$$
\mathrm{TOF}_{i, l}=\mathrm{TOF}_{\text {raw }, i, l}-a_{i, l}-\mathrm{TOF}_{\mathrm{MC}}^{\prime}
$$

For each data sample, the calibration procedure was applied to all the positions that were well-calibrated in terms of energy (see Sec. 2.4). In those positions the calibrated TOF was defined as the mean value between the calibrated front and rear TOF:

$$
\mathrm{TOF}_{i}=\frac{\mathrm{TOF}_{i, F}+\mathrm{TOF}_{i, R}}{2}
$$

\subsection{TOF resolution}

Using the TOF distributions obtained after the calibration, it was possible to assess the time resolution of the system. For each calibration sample, the overall TOF resolution was extracted by merging the data obtained from all the well-calibrated positions into one global distribution. For each sample, a Gaussian fit was applied to extract the mean value $\mu$ (TOF) and standard deviation $\sigma(\mathrm{TOF})$ of the distribution. This last parameter represents the TOF resolution of the system for the corresponding beam.

We then proceeded to remove the contribution of the intrinsic TOF fluctuations to the resolution by quadratically subtracting the spread observed in the un-tuned MC distributions. The extracted resolution $\sigma_{\text {par }}$ (TOF) was then parameterized as a function of the energy loss using the formula

$$
\sigma_{p a r}(\mathrm{TOF})=\sqrt{\frac{A}{\mu(\Delta E)}+B}
$$

where $A$ and $B$ are free parameters [16]. The resolution obtained from Eq. 11 was then used to produce the tuned MC simulations. As already done for $\Delta E$ values (see Sec. 2.5), the parameterization of the TOF resolution was performed considering only the CNAO samples.

We also assessed the individual contribution of the $\mathrm{SC}$ and the $\mathrm{TW}, \sigma_{\mathrm{SC}}(\mathrm{TOF})$ and $\sigma_{\mathrm{TW}}(\mathrm{TOF})$ respectively, to the overall TOF resolution by supposing that:

$$
\sigma^{2}(\mathrm{TOF})=\sigma_{\mathrm{TW}}^{2}(\mathrm{TOF})+\sigma_{\mathrm{SC}}^{2}(\mathrm{TOF})
$$

The TW contribution could be extracted from the distribution of the time difference between hits in front and rear layer in the same event (thus not using SC), fitting it with a Gaussian and extracting its standard deviation $\sigma_{\text {diff }}(\mathrm{TOF})$ in data. Then, noting that $\sigma_{\text {diff }}(\mathrm{TOF})$ corresponds to the quadratic sum of the time resolutions of each TW layer and considering that the error on $\Delta_{C L K}$ was negligible ( $\sim 5$ ps, see Sec. 3.2), error propagation of Eqs. 10, 8, 5 and 6 gave

$$
\sigma_{\mathrm{TW}}(\mathrm{TOF})=\frac{\sigma_{\text {diff }}(\mathrm{TOF})}{2}
$$

The contribution of the SC could then be derived from Eq. 12. 


\subsection{Charge identification}

Given the measured energy loss (Eq. 4) and TOF (Eq. 10), the charge $Z_{i}$ of a particle impinging on the TW in position $i$ was calculated for each event by inverting the Bethe-Bloch formula [27]:

$$
\beta=\frac{d}{c \cdot \mathrm{TOF}_{i}} \rightarrow Z_{i}=\sqrt{\frac{\Delta E_{i}}{\rho \delta x} \frac{\beta^{2} A_{S}}{K Z_{S}}\left(\frac{1}{2} \log \frac{2 m_{e} c^{2} \beta^{2} \gamma^{2} W_{\max }}{I^{2}}-\beta^{2}\right)^{-1}}
$$

Here $d$ is the distance traveled by the particles; $K=0.307 \mathrm{MeV} \cdot \mathrm{cm}^{2} / \mathrm{mol} ; \delta x=6 \mathrm{~mm}$ is the thickness of the two TW layers; $c$ is the speed of light; $\gamma=1 / \sqrt{\left(1-\beta^{2}\right)} ; m_{e} c^{2}=0.511 \mathrm{MeV}$ is the electron rest mass; $W_{\max }=2 m_{e} c^{2} \beta^{2} \gamma^{2}$ is the maximum energy transferred to an electron in one collision; and $\rho=1.023 \mathrm{~g} / \mathrm{cm}^{3}, Z_{S} / A_{S}=0.5417$ and $I=64.7 \mathrm{eV}$ are the density, the effective ratio of atomic and mass number and the mean excitation potential of the scintillator material, respectively [28]. Note that the Bethe-Bloch equation for describing the mean rate of energy loss is accurate up to a few \% for the beam energies considered [27].

For each calibration sample of Table 1, Eq. 14 was applied to all the events occurring in a TW position that was well-calibrated (see Sec. 2.4 and 2.6), obtaining the $Z$ spectrum of the beam. The $Z$ resolution was extracted by fitting the resulting spectrum with a Gaussian.

Concerning the fragmentation sample, an additional event selection was performed before applying Eq. 14. In each event, hit positions were identified by finding the crossings of the triggered bars in the two layers. If a nuclear reaction occurs, it is possible for two or more fragments to reach the TW at almost the same time, switching on multiple bars in each layer. In this case, the crossings of front and rear bars identify more positions than those actually hit by the fragments. To exclude the positions where the particles did not pass at all ('ghost hits'), we selected only those where $\Delta E_{i, F}$ and $\Delta E_{i, R}$ were similar:

$$
\frac{\left|\Delta E_{i, F}-\Delta E_{i, R}\right|}{\Delta E_{i}}<0.1
$$

The same selection cut was applied to the MC simulations.

\section{Results}

\subsection{Energy calibration and resolution}

Table 2 shows the $\mu$ and $\sigma$ values of the global $\Delta E$ distributions fitted with a Gaussian for the untuned MC simulations and for the data after they were calibrated. By comparing the fifth and third column in Table 2, we see that the mean values of the data distributions are indeed close to the reference mean values of the MC distributions. For protons, a relatively large difference was found. Apparently, the simple Birks-like model was not able to accurately describe energy losses all the way down to low $\Delta E$ values, mainly relevant for low $Z$ particles. This is not a problem, since the electronic setup of FOOT is not designed to perform precise measurements for such particles (see Sec. 1).

Moreover, we observe that the values for the energy resolution of the $\mathrm{MC}$ distribution (fourth column) are only about 1-3\%, coming purely from intrinsic fluctuations, while for the data (sixth column) we found energy resolutions from 4 to $6 \%$. The four values given in the last column for the CNAO data acquisitions were parameterized as $\sigma_{p a r}(\Delta E) / \mu(\Delta E) \approx 4.16 \%$. This parameterization was used to smear the energy loss and produce the tuned MC simulations. 
Table 2: Mean and sigma values obtained from Gaussian fits of the calibrated energy distributions for untuned MC simulations and data. The extracted resolution to be parameterized into the MC simulations is also given. Errors are not displayed, but fit errors on $\mu(\Delta E)$ and $\sigma(\Delta E) / \mu(\Delta E)$ for $\mathrm{MC}$ are below $0.001 \mathrm{MeV}$ and $0.01 \%$, respectively. Fit errors on $\mu(\Delta E)$ and $\sigma(\Delta E) / \mu(\Delta E)$ in data are below $0.07 \mathrm{MeV}$ and $0.04 \%$, respectively.

\begin{tabular}{|c|c|c|c|c|c|c|}
\hline \multicolumn{2}{|c|}{ Data sample } & \multicolumn{2}{c|}{$\mathrm{MC}$} & \multicolumn{2}{c|}{ Data } & For parameterization \\
\hline Particle & $\begin{array}{c}E_{\text {beam }} \\
{[\mathrm{MeV} / \mathrm{u}]}\end{array}$ & $\begin{array}{c}\mu(\Delta E) \\
{[\mathrm{MeV}]}\end{array}$ & $\begin{array}{c}\sigma(\Delta E) / \mu(\Delta E) \\
{[\%]}\end{array}$ & $\begin{array}{c}\mu(\Delta E) \\
{[\mathrm{MeV}]}\end{array}$ & $\begin{array}{c}\sigma(\Delta E) / \mu(\Delta E) \\
{[\%]}\end{array}$ & $\begin{array}{c}\sigma_{\text {par }}(\Delta E) \\
{[\%]}\end{array}$ \\
\hline $\mathrm{p}$ & 60 & 7.12 & 3.43 & 6.27 & 5.72 & 4.58 \\
${ }^{12} \mathrm{C}$ & 115 & 161.1 & 0.96 & 160.7 & 4.00 & 3.88 \\
${ }^{12} \mathrm{C}$ & 260 & 85.77 & 1.86 & 85.12 & 4.73 & 4.36 \\
${ }^{12} \mathrm{C}$ & 400 & 67.29 & 2.50 & 68.58 & 4.70 & 3.98 \\
\hline${ }^{16} \mathrm{O}$ & 400 & 119.9 & 1.91 & 120.5 & 5.19 & 4.82 \\
\hline
\end{tabular}

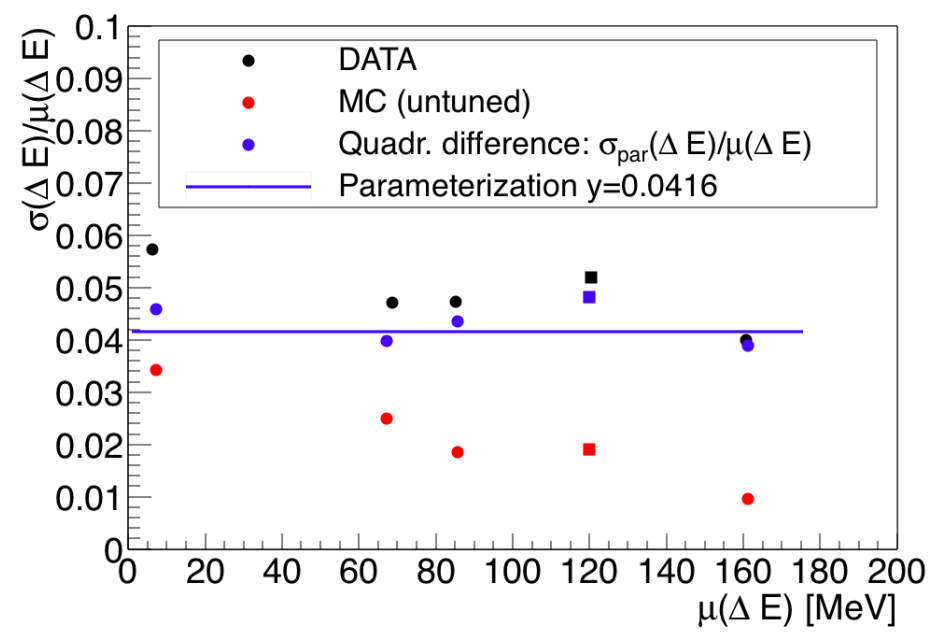

Figure 7: Energy resolution results extracted from data (black), untuned MC simulations (red) and the values obtained for $\sigma_{\text {par }}(\Delta E) / \mu(\Delta E)$ (blue). Also shown is the parameterization curve used for the Gaussian smearing of tuned MC simulations. The circle and square markers refer to the CNAO and GSI setup, respectively.

The data reported in Tab. 2 and the resulting parameterization used to produce the tuned MC simulations are showed in Fig. 7.

In Fig. 8 we show two examples (one for CNAO and one for GSI) of the energy spectrum obtained in data and tuned MC simulations. Looking at the GSI energy spectrum, we note that the width of the spectrum is slightly larger in data than in tuned MC simulations. This is because the resolution parameterization was based on CNAO data, i.e., under different experimental circumstances and with a different type of particle (see Sec. 4).

\subsection{TOF calibration and resolution}

In Table 3 we give the $\mu$ and $\sigma$ values of TOF distributions fitted with a Gaussian for the $\mathrm{MC}$ simulations and for the data after they were calibrated.

The resolution in data $\left(\sigma(\mathrm{TOF})\right.$, sixth column) was varying from $54 \mathrm{ps}\left(115 \mathrm{MeV} / \mathrm{u}^{12} \mathrm{C}\right)$ to $265 \mathrm{ps}$ (protons). Note that, in the case of TOF, the contribution of the intrinsic term to the overall resolution is always negligible. Thus, we have that $\sigma_{p a r}(\mathrm{TOF})=\sigma(\mathrm{TOF})$. With these values, the parameters extracted from the fit in Eq. 11 were $A=(3.72 \pm 0.02) \cdot 10^{-1} \mathrm{~ns}^{2} / \mathrm{MeV}^{2}$ 


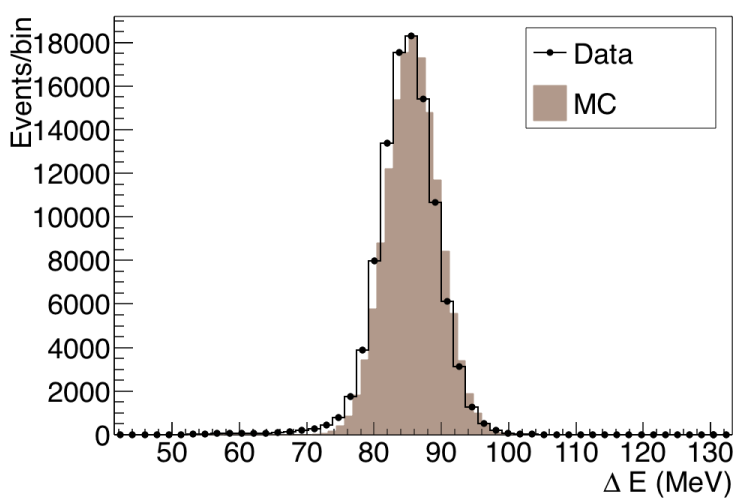

(a)

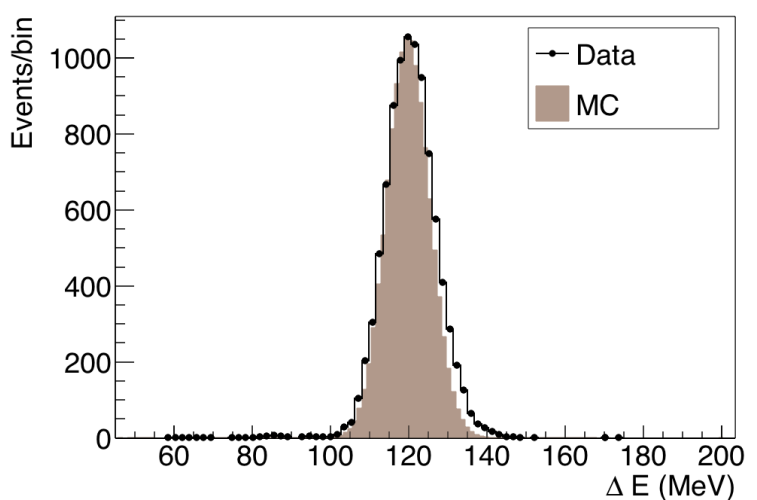

(b)

Figure 8: (a) Energy spectrum for the CNAO calibration run with $260 \mathrm{MeV} / \mathrm{u}{ }^{12} \mathrm{C}$ ions, for calibrated data (black) and tuned MC (brown). (b) The same, but for $400 \mathrm{MeV} / \mathrm{u}{ }^{16} \mathrm{O}$ ions at GSI.

Table 3: Mean and sigma values obtained from Gaussian fits of the calibrated TOF distributions for untuned MC simulations and data. The extracted values for resolution parameterization are also given. Uncertainties are not displayed, but fit errors on $\mu$ (TOF) and $\sigma(\mathrm{TOF})$ are below 1 ps for both $\mathrm{MC}$ and data.

\begin{tabular}{|c|c|c|c|c|c|c|}
\hline \multicolumn{2}{|c|}{ Data sample } & \multicolumn{2}{|c|}{ MC } & \multicolumn{2}{c|}{ Data } & For parameterization \\
\hline Particle & $\begin{array}{c}E_{\text {beam }} \\
{[\mathrm{MeV} / \mathrm{u}]}\end{array}$ & $\begin{array}{c}\mu(\mathrm{TOF}) \\
{[\mathrm{ns}]}\end{array}$ & $\begin{array}{c}\sigma(\mathrm{TOF}) \\
{[\mathrm{ns}]}\end{array}$ & $\begin{array}{c}\mu(\mathrm{TOF}) \\
{[\mathrm{ns}]}\end{array}$ & $\begin{array}{c}\sigma(\mathrm{TOF}) \\
{[\mathrm{ns}]}\end{array}$ & $\begin{array}{c}\sigma_{\text {par }}(\mathrm{TOF}) \\
{[\mathrm{ns}]}\end{array}$ \\
\hline $\mathrm{p}$ & 60 & 4.207 & 0.003 & 4.207 & 0.265 & 0.265 \\
${ }^{12} \mathrm{C}$ & 115 & 3.205 & 0.001 & 3.204 & 0.054 & 0.054 \\
${ }^{12} \mathrm{C}$ & 260 & 2.291 & 0.001 & 2.291 & 0.066 & 0.066 \\
${ }^{12} \mathrm{C}$ & 400 & 1.997 & 0.001 & 1.996 & 0.074 & 0.074 \\
\hline${ }^{16} \mathrm{O}$ & 400 & 10.449 & 0.001 & 10.447 & 0.084 & 0.084 \\
\hline
\end{tabular}

and $B=(4.0 \pm 0.2) \cdot 10^{-4} \mathrm{~ns}^{2}$. The results reported in Tab. 3 and the parameterization curve used to produce the tuned MC simulations are shown in Fig. 9.

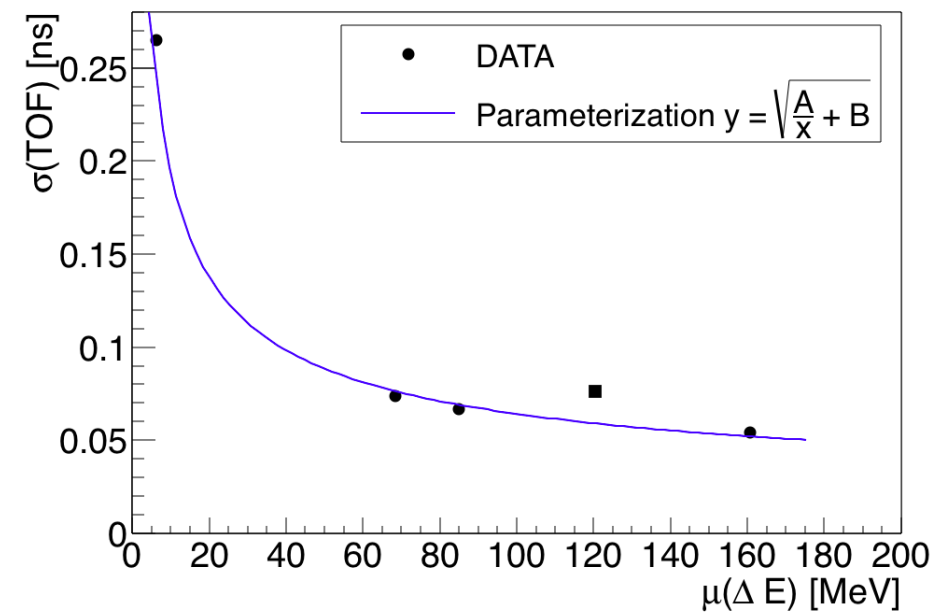

Figure 9: TOF resolution results (black) and corresponding parameterization curve (blue) used to produce the tuned MC simulations. The circle and square marker refers to the CNAO and GSI setup, respectively. 
In Fig. 10 we show two examples for the calibrated TOF spectra, one for CNAO (10a) and one for GSI (10b). The corresponding spectra for the tuned MC simulations are also shown. Concerning the CNAO samples, data were seen to match well with the MC simulations.

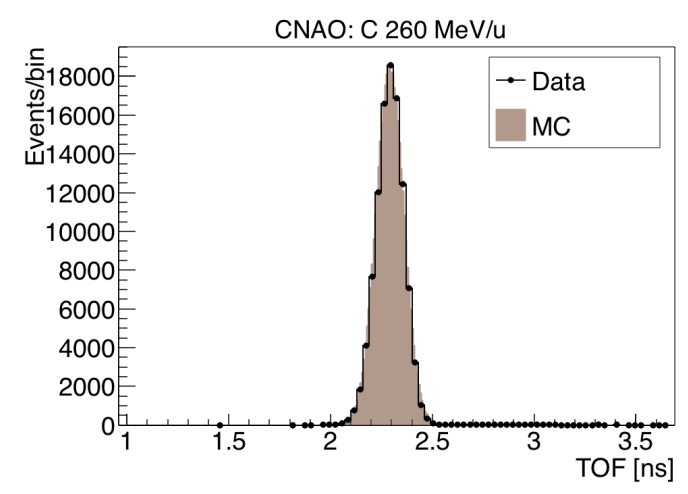

(a)

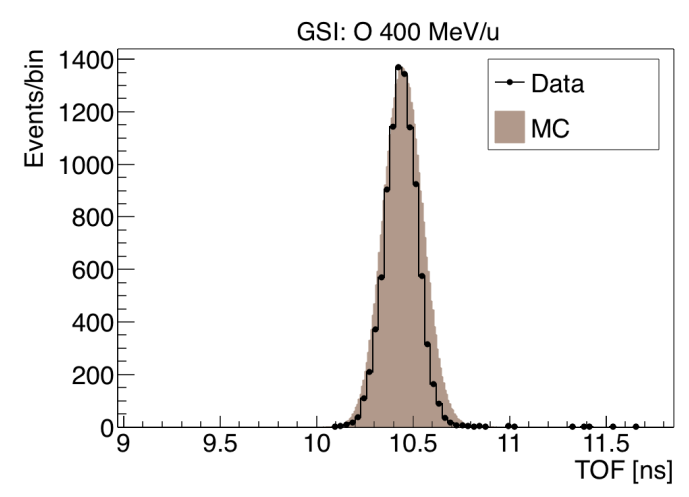

(b)

Figure 10: (a) TOF data spectra for the CNAO calibration run with $260 \mathrm{MeV} / \mathrm{u}^{12} \mathrm{C}$ ions, for calibrated data (black) and tuned MC simulations (brown). (b) The same, but for $400 \mathrm{MeV} / \mathrm{u}^{16} \mathrm{O}$ ions at GSI.

Instead, the GSI data (Fig. 10b) had a slightly larger width than the MC simulation, which can be explained by the differences in the experimental setup. In fact, the distance between the SC and the TW at GSI was much larger and other FOOT components were present, so that a parameterization based on CNAO data may not be accurate to estimate the exact behaviour of the experimental resolution at GSI.

Table 4 shows the contributions to the time resolution of the individual detectors. As expected, the TOF resolution is dominated by the $\mathrm{SC}$ because of its reduced thickness with respect to TW bars.

Table 4: TOF resolution of the system and relative contributions from single detectors. The results refer to all positions summed together. Errors represent fit errors.

\begin{tabular}{|c|c|c|c|c|}
\hline Particle & $\begin{array}{c}E_{\text {beam }} \\
{[\mathrm{MeV} / \mathrm{u}]}\end{array}$ & $\begin{array}{c}\sigma(\mathrm{TOF}) \\
{[\mathrm{ps}]}\end{array}$ & $\begin{array}{c}\sigma_{\mathrm{TW}}(\mathrm{TOF}) \\
{[\mathrm{ps}]}\end{array}$ & $\begin{array}{c}\sigma_{\mathrm{SC}}(\mathrm{TOF}) \\
{[\mathrm{ps}]}\end{array}$ \\
\hline $\mathrm{p}$ & 60 & $264.9 \pm 0.8$ & $85.3 \pm 0.2$ & $250.8 \pm 0.4$ \\
${ }^{12} \mathrm{C}$ & 115 & $54.4 \pm 0.1$ & $19.7 \pm 0.1$ & $50.7 \pm 0.1$ \\
${ }^{12} \mathrm{C}$ & 260 & $66.4 \pm 0.2$ & $22.8 \pm 0.1$ & $62.4 \pm 0.1$ \\
${ }^{12} \mathrm{C}$ & 400 & $73.6 \pm 0.2$ & $24.7 \pm 0.1$ & $69.4 \pm 0.1$ \\
\hline${ }^{16} \mathrm{O}$ & 400 & $83.9 \pm 0.7$ & $42.0 \pm 0.3$ & $72.6 \pm 0.6$ \\
\hline
\end{tabular}

\subsection{Charge identification}

In Table 5 we list the $\mu$ and $\sigma$ values for the $Z$ distribution in data. In all cases, the reconstructed $Z$ values were within a few percent of the expected charge of the primary beams. For ${ }^{12} \mathrm{C}$ and ${ }^{16} \mathrm{O}$ the obtained resolutions are compatible with the overall requirements of the experiment (see Sec. 1).

The extracted calibration parameters were also used to analyze the fragmentation run performed at GSI with ${ }^{16} \mathrm{O}$ ions on a $5 \mathrm{~mm}$ graphite target. The fragmentation spectrum is shown in Fig. 11. This figure shows that the differently charged fragments can be well discriminated. 
Table 5: Mean and sigma values obtained from Gaussian fits of the $Z$ distributions for data. Uncertainties are not displayed, but the fit errors on $\mu(Z)$ and $\sigma(Z)$ were below 0.002 .

\begin{tabular}{|c|c|c|c|c|}
\hline Particle & $E_{\text {beam }}[\mathrm{MeV} / \mathrm{u}]$ & $\mu(Z)$ & $\sigma(Z)$ & $\sigma(Z) / \mu(Z)[\%]$ \\
\hline $\mathrm{p}$ & 60 & 0.96 & 0.06 & $6.10 \pm 0.02$ \\
${ }^{12} \mathrm{C}$ & 115 & 6.17 & 0.15 & $2.51 \pm 0.01$ \\
${ }^{12} \mathrm{C}$ & 260 & 6.01 & 0.21 & $3.52 \pm 0.01$ \\
${ }^{12} \mathrm{C}$ & 400 & 6.07 & 0.24 & $3.85 \pm 0.01$ \\
\hline${ }^{16} \mathrm{O}$ & 400 & 8.07 & 0.22 & $2.67 \pm 0.02$ \\
\hline
\end{tabular}

\section{Fragmentation run GSI: O $400 \mathrm{MeV} / \mathrm{u}$}

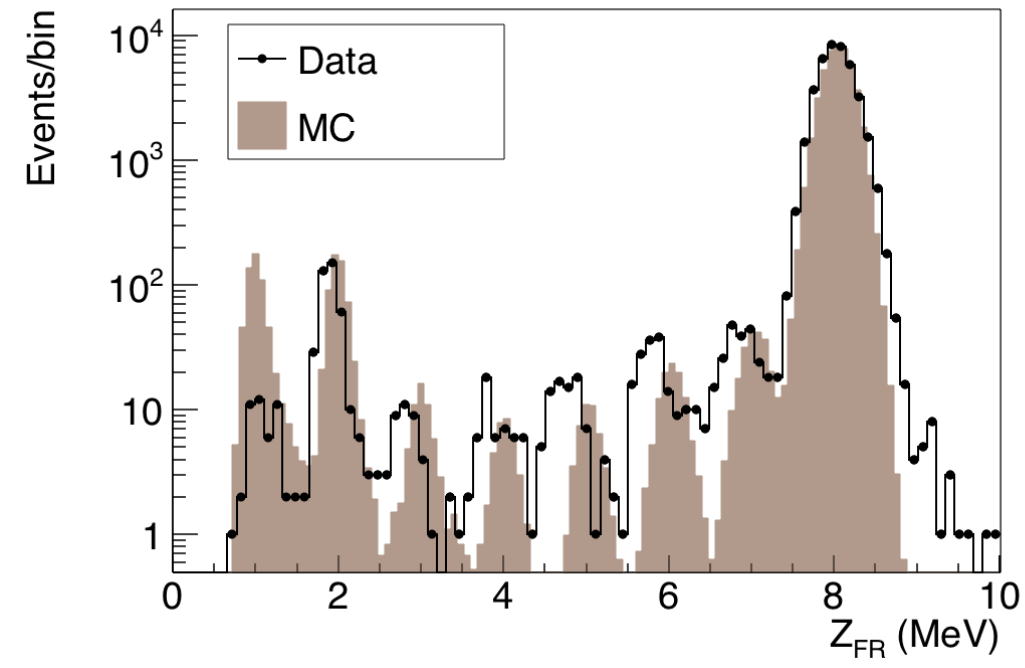

Figure 11: $Z$ spectrum obtained from the fragmentation data of ${ }^{16} \mathrm{O}$ ions on graphite at GSI.

\section{Discussion}

In this work, we developed and tested a calibration method to extract the values for TOF and $\Delta E$ which are needed for charge discrimination. We also assessed the detector performances to discriminate the charge of fragments. The method was based on a position-by-position calibration with MC reference values. The advantage of the approach is that it is robust and precise. In fact, even if variations are present between the individual bars, for instance due to differences in optical coupling, SiPM gain, total internal reflection efficiency or possible issues in WDBs, the response of the detector could still be modeled accurately. One of the disadvantages of this method is that the TW needs to be irradiated in all the positions, meaning that enough beam time should be dedicated to the calibration of the $\Delta E$-TOF when planning future data takings. A more elegant solution would be to investigate position dependence in the signals of the bars. Work is ongoing to improve the modeling of all the effects currently not accounted for in the MC simulations, including transport of optical photons inside the bars and SiPM response.

We found various issues that should be improved and investigated in-depth:

- The combination of two different experimental setups for calibration and fragmentation runs is complex and should be avoided. Small incompatibilities are expected in such situations. For future data-takings, it is better to calibrate and acquire fragmentation data under exactly the same experimental conditions.

- We noticed that the gains in the SiPMs were not fully stable, even within the same 
experimental data setup. In particular, in various bars variations were observed in mean charge up to about $3 \%$ between runs at GSI. Sensors will be added in order to monitor possible fluctuations in temperature that can affect SiPM signals.

- The light output model should be studied in more detail. The response of many organic scintillators varies with particle type (see e.g. [29]). Using a single curve based on the most simple Birks' model to describe the collected charge of three types of particles ( $\mathrm{p}$, $\mathrm{C}$ and $\mathrm{O}$ ) over a large energy loss range (from 2 to $90 \mathrm{MeV}$ on a $3 \mathrm{~mm}$ bar) may not be fully appropriate. Several other models were tested, such as the halo model [29], but they did not significantly improve the results. New data with more types of ions over a wider energy range will be acquired in 2021, so that the light output model can be studied in detail.

- The mechanical stability of the detector should be improved. A new prototype is under construction, based on the same bars, but with a more stable frame. With this new setup, the optical isolation between the bars will also be improved. This setup will be tested at the end of 2020 .

Despite the above issues, we demonstrated that TW and SC detectors together with the new DAQ system were able to discriminate the fragment charges.

\section{Conclusions}

In this work, we proposed a charge identification procedure of nuclear fragments for the first full-scale prototype of the Time-Of-Flight system of FOOT. The obtained results show that the developed $\Delta E$ and TOF calibration procedures are effective and reproduce the MC distributions with good accuracy. Even though it is under development, the system showed promising performances in terms of both $\Delta E$ (3.9-5.3\%) and TOF (50-75 ps for heavy ions, $250 \mathrm{ps}$ for protons) resolution in view of future acquisitions with the full FOOT setup. The final resolution on charge measurements is compatible with the requirements of the experiment, ranging from about $6.2 \%$ for the proton beam to $2.5 \%$ for ${ }^{12} \mathrm{C}$ and ${ }^{16} \mathrm{O}$ ions.

The $Z$ identification procedure has been applied for the first time with the FOOT detector to study the nuclear fragments produced by the irradiation of a graphite target with a ${ }^{16} \mathrm{O}$ beam, showing that the system is already able to discriminate different charged particles with good accuracy.

\section{Acknowledgements}

The FOOT Collaboration acknowledges the INFN for its support in building and running the detector. We would like to acknowledge all the personnel of the CNAO and GSI centres that provided us support during the operational tests performed using proton, ${ }^{12} \mathrm{C}$ and ${ }^{16} \mathrm{O}$ beams at their facilities. The GSI data were taken in the IBER 006 experiment, supported by the European Space Agency (ESA) - IBER17 717 project, in the frame of FAIR Phase-0.

\section{References}

[1] M. Durante and H. Paganetti. "Nuclear Physics in Particle Therapy: a review". In: Reports on Progress in Physics 79.9 (2016), p. 096702. url: https://dx.doi.org/10.1088\% 2F0034-4885\%2F79\%2F9\%2F096702. 
[2] F. Tommasino and M. Durante. "Proton Radiobiology". In: Cancers 7.1 (2015), pp. 353381. URL: https://dx.doi.org/10.3390/cancers7010353.

[3] C. K. Ying, D. Bolst, L. T. Tran, et al. "Contributions of secondary fragmentation by carbon ion beams in water phantom: Monte Carlo simulation". In: J. Phys.: Conference Series 851 (2017), p. 012033. URL: https://dx.doi.org/10.1088\%2F1742-6596\%2F851\% $2 \mathrm{~F} 1 \% 2 \mathrm{~F} 012033$.

[4] Osama M., J. B. Sishc, J. Saha, et al. "Carbon Ion Radiotherapy: A Review of Clinical Experiences and Preclinical Research, with an Emphasis on DNA Damage/Repair". In: Cancers 9.6 (2017), p. 66. URL: https://dx.doi.org/10.3390/cancers9060066.

[5] J. S. Loeffler and M. Durante. "Charged particle therapy-optimization, challenges and future directions". In: Nat Rev Clin Oncol 10 (7 2013), pp. 411-424. URL: https://dx. doi.org/10.1038/nrclinonc.2013.79.

[6] S. Muraro, G. Battistoni, and A.C. Kraan. "Challenges in Monte Carlo simulations as clinical and research tool in particle therapy: a review". In: Front. Phys. (2020). (Accepted for publication). URL: https://dx.doi.org/10.3389/fphy.2020.567800.

[7] M. De Napoli, C. Agodi, G. Battistoni, et al. "Carbon fragmentation measurements and validation of the GEANT4 nuclear reaction models for hadron therapy". In: Phys. Med. Biol. 57.22 (2012), pp. 7651-71. URL: https://dx.doi.org/10.1088/0031-9155/57/22/ 7651.

[8] J. Dudouet, D. Juliani, M. Labalme, et al. "Double-differential fragmentation cross-section measurements of $95 \mathrm{MeV} /$ nucleon ${ }^{12} \mathrm{C}$ beams on thin targets for hadron therapy". In: Phys. Rev. C 88 (2 2013), p. 024606. URL: https://dx.doi.org/10.1103/PhysRevC. 88.024606 .

[9] M. Toppi, Z. Abou-Haidar, C. Agodi, et al. "Measurement of fragmentation cross sections of ${ }^{12} \mathrm{C}$ ions on a thin gold target with the FIRST apparatus". In: Phys. Rev. C 93 (6 2016), p. 064601. URL: https://dx.doi.org/10.1103/PhysRevC.93.064601.

[10] I. Mattei, A. Alexandrov, and L. Alunni Solestizi. "Measurement of ${ }^{12} \mathrm{C}$ Fragmentation Cross Sections on C, O, and H in the Energy Range of Interest for Particle Therapy Applications". In: IEEE Transactions on Radiation and Plasma Medical Sciences 4.2 (2020), p. 269. URL: https://dx.doi.org/10.1109/TRPMS. 2020.2972197.

[11] L. Walsh, U. Schneider, A. Fogtman, et al. "Research plans in Europe for radiation health hazard assessment in exploratory space missions". In: Life Sciences in Space Research 21 (2019), pp. 73-82. URL: https://dx.doi.org/10.1016/j.Issr.2019.04.002.

[12] G. Battistoni, M. Toppi, A. Alexandrov, et al. "Measuring the impact of Nuclear Interaction in Particle Therapy and in Radio Protection in Space: the FOOT experiment". In: Front. Phys. 8 (2021), p. 555. DOI: https://doi.org/10.3389/fphy.2020.568242.

[13] FOOT collaboration. FOOT Conceptual Design Report. 2017.

[14] S. M. Valle. "Design, simulation and performances study of the FOOT experiment". PhD Thesis. Università degli studi di Milano, 2019. URL: http://dx . doi .org/10 . 13130/ valle-serena-marta_phd2019-01-18.

[15] G. Traini, A. Alexandrov, B. Alpat, et al. "Performance of the ToF detectors in the FOOT experiment". In: Il Nuovo Cimento (2020). URL: https://dx.doi.org/10.1393/ncc/ i2020-20016-5. 
[16] M. Morrocchi, E. Ciarrocchi, A. Alexandrov, et al. "Development and characterization of a $\triangle \mathrm{E}-\mathrm{TOF}$ detector prototype for the FOOT experiment". In: Nucl. Instrum. Methods Phys. Res. A 916 (2019), pp. 116-124. URL: https://dx.doi.org/10.1016/j.nima. 2018.09.086.

[17] E. Ciarrocchi, N. Belcari, N. Camarlinghi, et al. "The $\triangle \mathrm{E}-\mathrm{TOF}$ detector of the FOOT experiment: Experimental tests and Monte Carlo simulations". In: Nucl. Instrum. Methods Phys. Res. A 936 (2019), pp. 78-79. URL: https://dx.doi.org/10.1016/j.nima.2018. 08.117.

[18] L. Galli, A.C. Kraan, E. Ciarrocchi, et al. "Fragment charge identification technique with a plastic scintillator detector using clinical carbon beams". In: Nucl. Instrum. Methods Phys. Res. A 953 (2020), p. 163146. URL: https://dx.doi.org/10.1016/j.nima.2019.163146.

[19] A.C. Kraan, G. Battistoni, N. Belcari, et al. "Charge identification performance of a $\Delta \mathrm{E}-$ TOF detector prototype for the FOOT experiment". In: Nucl. Instrum. Methods Phys. Res. A 958 (2020), p. 162422. URL: https://dx.doi.org/10.1016/j.nima.2019.162422.

[20] S. Ritt, R. Dinapoli, and U. Hartmann. "Application of the DRS chip for fast waveform digitizing". In: Nucl. Instrum. Methods Phys. Res. A 623.1 (2010), pp. 486-488. URL: https://dx.doi.org/10.1016/j.nima.2010.03.045.

[21] S. Ritt. "The DRS chip: cheap waveform digitizing in the GHz range". In: Nucl. Instrum. Methods Phys. Res. A 518.1 (2004), pp. 470-471. URL: https://dx.doi.org/10.1016/ j.nima.2003.11.059.

[22] L. Galli, A.M. Baldini, F. Cei, et al. "WaveDAQ: An highly integrated trigger and data acquisition system". In: Nucl. Instrum. Methods Phys. Res. A 936 (2019), pp. 399-400. URL: https://dx.doi.org/10.1016/j.nima.2018.07.067.

[23] G. Battistoni, J. Bauer, T. T. Boehlen, et al. "The FLUKA Code: an Accurate Simulation Tool for Particle Therapy". In: Front. Oncol. 6 (2016), p. 116. URL: https://dx. doi . org/10.3389/fonc.2016.00116.

[24] A. Ferrari, P. R. Sala, A. Fassò, and J. Ranft. "FLUKA: a multi-particle transport code". In: (2018). URL: http://www.fluka.org/content/manuals/online/INDEX-fluka2020. html.

[25] J. B. Birks. The Theory and Practice of Scintillation Counting. International Series of Monographs in Electronics and Instrumentation. Pergamon, 1964, pp. xvii-xviii. ISBN: 978-0-08-010472-0. URL: https://dx.doi.org/10.1016/C2013-0-01791-4.

[26] R. Zarrella. "Charge identification of nuclear fragments with the Time-Of-Flight detectors of the FOOT experiment". Master Thesis. Università degli studi di Pisa, 2020. URL: https : / / etd . adm . unipi . it/theses / available / etd-03162020-114207 / unrestricted / Thesis_Roberto_Zarrella.pdf.

[27] Particle data group. Passage of particles through matter. 2009. URL: http://pdg. Ibl. gov/2009/reviews/rpp2009-rev-passage-particles-matter.pdf.

[28] Particle Data Group (PDG). Atomic and nuclear properties of polyvinyltoluene. 2020. URL: https : / / pdg . lbl . gov / 2018/AtomicNuclearProperties / HTML / polyvinyltoluene . html. 
[29] P.S. Marrocchesi, O. Adriani, Y. Akaike, et al. "Beam test performance of a scintillatorbased detector for the charge identification of relativistic ions". In: Nucl. Instrum. Methods Phys. Res. A 659.1 (2011), pp. 477-483. uRL: https://dx.doi.org/10.1016/j.nima. 2011.08.034. 